\title{
Immediate Intravenous Epinephrine Versus Early Intravenous Epinephrine for In-Hospital Cardiopulmonary Arrest
}

Abdullah Bakhsh ( $\square$ aarbakhsh@kau.edu.sa )

King Abdulaziz University

Maha Safhi

King Abdulaziz University

Ashwaq Alghamdi

King Abdulaziz University

Amjad Alharazi

King Abdulaziz University

Bedoor Alshabibi

King Abdulaziz University

\section{Rajwa Alobaidi}

King Abdulaziz University

Maryam Alnashri

King Abdulaziz University

\section{Research Article}

Keywords: in-hospital cardiopulmonary arrest, immediate epinephrine, ROSC, survival to admission

Posted Date: February 4th, 2021

DOl: https://doi.org/10.21203/rs.3.rs-153455/v1

License: (c) (i) This work is licensed under a Creative Commons Attribution 4.0 International License. Read Full License 


\section{Abstract \\ Background}

intravenous epinephrine has been a key treatment for cardiopulmonary arrest since the early 1960s. Although, many studies have questioned neurological outcome benefit, it remains to be recommended in international guidelines for its benefit on return of spontaneous circulation (ROSC). The ideal timing for the first epinephrine dose is uncertain. We aimed to look at the association of immediate epinephrine administration (within 1-minute of cardiac arrest recognition) with return of spontaneous circulation (ROSC) up to 24-hours and beyond 24-hours.

\section{Methods}

this was a multicenter retrospective chart review of patients undergoing cardiopulmonary resuscitation. Descriptive statistics were used to characterize study population, while t-test and chi-square were used to compare groups and outcomes.

\section{Results}

immediate epinephrine administration (within 1-minute) is associated with higher rates of ROSC up to 24hours $(\mathrm{OR}=2.36,95 \% \mathrm{Cl} ;[1.46-3.81])$ and beyond 24-hours $(\mathrm{OR}=2.26,95 \% \mathrm{Cl}$; $[1.06-4.83])$.

\section{Conclusions}

we encourage immediate administration of epinephrine in conjunction with high-quality CPR, as this is associated with higher rates of ROSC.

\section{Introduction}

Epinephrine has been a key treatment of advanced cardiac life support (ACLS) since cardiopulmonary resuscitation (CPR) guidelines were first published in early 1960s. [1] Epinephrine's alpha-agonist effect causes increased aortic diastolic pressure, thereby augmenting coronary blood flow and cerebral blood flow. [1] Various studies have shown that Epinephrine is associated with increased return of spontaneous circulation (ROSC) rates, owing to the alpha-agonistic effects. [2,3] However, there is uncertainty about its effect on survival to hospital discharge and neurologic recovery. $[4,5,6]$ On the other hand, Epinephrine may produce a mismatch between oxygen demand and delivery which results in lactic acidosis. Moreover, the vasoconstrictor effects may prolong ischemia in some tissues. This has been seen particularly in Swine brain. $[7,8]$ In fact, direct visualization of brain capillaries reveal constricted microvessels, with little or no perfusion to brain tissue. This effect was attributable to the alpha-1 agonist effects of Epinephrine. $[1,7,8]$ 
Goto et al, looked at the pre-hospital use of intravenous Epinephrine and its effect on return of spontaneous circulation and neurological outcomes. When given within 9-minutes of cardiac arrest, Epinephrine is associated with higher rates of return of spontaneous circulation (ROSC), when compared with patients not receiving Epinephrine. Neurologic outcomes, however were poorer in patients receiving Epinephrine at any given time during cardiac arrest. [5]

The American Heart Association (AHA) recommends administering Epinephrine as early as feasibly possible and thereafter, every 3-5 minutes. [9] Various trials suggest a time-dependent effect of Epinephrine on outcomes of CPR; earlier administration of intravenous Epinephrine may improve outcomes. $[10,11]$ However, previous studies have shown delays in the administration of epinephrine are common in clinical practice, and thus found to be associated with worse outcomes in both adults and children. $[12,13]$

A study in 2014 showed that earlier administration of epinephrine in patients with non-shockable cardiac arrest rhythms are associated with increased ROSC and survival. Moreover, a stepwise decrease in survival with every increase in interval of time to epinephrine. [12] Another study in 2016 found that there were improvements in ROSC and survival with functional recovery with timely administration of Epinephrine. [14] Recently, in 2019 a study revealed that delays in intravenous epinephrine administration was associated with lower survival. [15]

The lack of rigorous experimental studies on the clinical outcomes associated with epinephrine has led the resuscitation community to continue to recommend its use in cardiac arrest. However, the PARAMEDIC-2 trial might change the way clinicians think about Epinephrine. The study, conducted by Perkins et al. in the United Kingdom, included 8,014 patients with out-of-hospital cardiac arrest. Patients were randomized to receive either epinephrine $(n=4,015)$ or placebo $(n=3,999)$. Primary outcome was 30-day survival, and secondary outcomes included survival to hospital discharge and neurologically intact status. The authors found that administration of epinephrine increased 30 -day survival rates (3.2\% in the epinephrine group, compared to $2.4 \%$ in the placebo group). However, a larger proportion of patients in the epinephrine group were neurologically devastated, with modified Rankin scores of 4-5 ( $31 \%$ in the epinephrine group, compared to $17.8 \%$ in the placebo group). This result explains the lack of overall improvement in neurologically intact survival in the epinephrine group, despite the higher rate of overall survival. $[16,17]$ The authors postulate that, despite improving macrovascular cerebral perfusion pressures, epinephrine may cause microvascular ischemia in the brain, thereby worsening anoxic brain injury. A key finding of the PARAMEDIC-2 trial is that the mean time to Epinephrine administration was 21.5 minutes. This dramatic different time frame precludes generalizability to the in-hospital setting.

Although Epinephrine can increase the likelihood of achieving ROSC, the optimal time of Epinephrine is still uncertain. [17] It seems intuitive that immediate administration of epinephrine in combination with cardiopulmonary resuscitation will maintain perfusion and therefore, reduce bad outcomes. Our primary objective is to compare the association of immediate administration of epinephrine (within 1-minute) 
with early administration of epinephrine ( $\geq 2$-minutes) with sustained ROSC ( $\geq 20$-minutes -24 -hours) and ROSC for more than 24-hours in non-shockable in-hospital cardiopulmonary arrest.

\section{Materials And Methods}

\subsection{Study design:}

This is a multicenter retrospective chart review at three Ministry of Health hospitals in the city of Jeddah: King Fahad General Hospital, King Abdulaziz Hospital (Al Mahjer) and Althagher Hospital. After obtaining IRB approval (registration no. H-02-J-002, approval no. A00440), researchers retrospectively collected data from cardiac arrest flowsheets from each site between January 2016 and January 2017. We included patients 1) $\geq 18$ years-old, 2) non-shockable rhythms, 2) received intravenous Epinephrine during cardiopulmonary resuscitation, 3) witnessed in-hospital arrest and 4) only the first resuscitation attempt (for patients requiring more than one attempt at resuscitation). We excluded patients suffering traumatic arrest, were pregnant, had shockable rhythms, in the operating room, had a Do-Not-Resuscitate (DNR) order, and aged 17 years-old or less.

\subsection{Study setting:}

It is standard procedure in the Kingdom of Saudi Arabia to activate a code blue announcement throughout the hospital to summon the resuscitation team. The agreed upon definition for cardiopulmonary arrest at each site is cessation of cardiac function manifested as a non-palpable carotid pulse. Upon recognition, personnel are required to call for assistance and immediately start chest compressions. Each site requires medical staff to be certified in basic life support at the minimum. Code blue team members are typically composed of a critical care physician, an anesthesiologist, an intensive care unit nurse, in addition to the primary team and staff nurse from the location of arrest. Team members responding to code blue activations are certified in advanced cardiac life support to ensure standardized treatment. The composition of the resuscitation team may differ from institution to another, based on staff expertise and patient needs. Cardiopulmonary arrest flow sheets may differ in format between institutions; however, all contain key information based on Utstein guideline for documentation. Data included on the flow sheet include: patient information and demographics, time and date of arrest, location of arrest, available team members and time of response, initial and subsequent rhythms, medications and doses administered, type of airway device placed, presence or absence of return of spontaneous circulation, and post-arrest vital signs. Data is entered in real time during the resuscitation by a nurse designated and trained in documentation.

\subsection{Definitions:}

Time to epinephrine was defined as the interval in minutes from recognition of loss of pulse to the first bolus dose of 1 milligram intravenous epinephrine. A registered nurse is dedicated for documenting time intervals during resuscitation in all resuscitations. Each center provide special training for documenting events during resuscitations to ensure standardization. 


\subsection{Statistical analysis:}

We used descriptive statistics to characterize the study population. We used t-test to compare the means of age and CPR duration between the 2 groups. We used chi square test to compare number of patients intubated between the 2 groups. Outcomes between the groups were compared using the chi square test. A p-value of less than 0.05 was considered significant. We conducted all analyses using Microsoft Excel 2016 data analysis ToolPak (Redmond, WA, USA).

\section{Results}

We screened a total of 589 charts from 3 different sites. After excluding patients less than 18 -years-old, pregnant women, traumatic cardiopulmonary arrests, cardiopulmonary arrests occurring in the operating room, shockable rhythms, and patients who did not receive intravenous Epinephrine, we were left with 345 patients for analysis. Table 1 shows the baseline characteristics of patients. The mean time to Epinephrine administration was 2.38 minutes ( \pm 5.08 minutes). Sustained return of spontaneous circulation (ROSC) (20-minutes - 24-hours) occurred in 105 patients (30.35\%); 47 (13.58\%) survived to hospital admission and $13(3.76 \%)$ survived to hospital discharge.

Immediate (within 1-minute) Epinephrine administration was more frequently observed in 211 patients (60.98\%), whereas early ( $\geq 2$-minutes) Epinephrine administration was seen in 135 patients (39.02\%), as shown in Table 2.

Our results reveal that the immediate administration of intravenous Epinephrine is associated with higher rates of ROSC (20-minutes - 24-hours); $36.67 \%$ vs. $19.87 \%$ (OR $=2.36,95 \% \mathrm{Cl}$; [1.46-3.81]), and higher rates of ROSC (>24-hours); $14.44 \%$ vs. $6.92 \%$ (OR $=2.26,95 \% \mathrm{Cl}$; [1.06-4.83]) (Table 3). Moreover, age, CPR duration and endotracheal intubation were similar between the 2 groups.

A graphical illustration (Fig. 1) shows a stepwise decrease in sustained ROSC (20-minutes - 24-hours) with every 1-minute delay in Epinephrine administration: $36.67 \%$ showed sustained ROSC when receiving their first dose of Epinephrine between 0-1 minute. This is decreased to $21.21 \%$ when the first dose of Epinephrine was received between 2-3 minutes, 21.21\% when Epinephrine received between 4 - 3 minutes and down to $13.79 \%$ when the first dose was administered at 6-minutes or later. Figure 2 also demonstrates a decrease in ROSC for than 24-hours when epinephrine was administered at 2-minutes or later. 
Table 1

Baseline characteristics of patients

\begin{tabular}{|c|c|}
\hline Characteristic & $\mathbf{N}(\%)$ \\
\hline \multicolumn{2}{|l|}{ Gender $(n=346)$} \\
\hline Male & $208(60.12 \%)$ \\
\hline Female & $138(39.88 \%)$ \\
\hline \multicolumn{2}{|l|}{ Race $(n=347)$} \\
\hline Saudi & $181(52.16 \%)$ \\
\hline Non-Saudi & $166(47.84 \%)$ \\
\hline Mean age in years $\pm(S D)$ & $61.42 \pm 18.94$ \\
\hline \multicolumn{2}{|l|}{ Initial rhythm ( $n=346)$} \\
\hline Asystole & $215(62.14 \%)$ \\
\hline Pulseless Electrical Activity (PEA) & $131(37.86 \%)$ \\
\hline \multicolumn{2}{|l|}{ Outcome $(n=346)$} \\
\hline ROSC (> 20-min - 24-hrs) & $105(30.35 \%)$ \\
\hline Survival to Admission (> 24-hrs) & $47(13.58 \%)$ \\
\hline Survival to Discharge & $13(3.76 \%)$ \\
\hline \multicolumn{2}{|l|}{ Mean Times $(n=346)$} \\
\hline Time to Epinephrine [mean in minutes $\pm(S D)]$ & $2.38 \pm(5.08)$ \\
\hline Duration of CPR [mean in minutes $\pm(S D)]$ & $19: 06 \pm(10: 12)$ \\
\hline
\end{tabular}

Table 2

Rate of Immediate Epinephrine (within 1-minute) Administration versus Early Epinephrine ( $\geq 2$ minutes) Administration

\begin{tabular}{|lll|}
\hline & N (346) & $\%$ \\
\hline Immediate (within 1-minute) & 211 & $60.98 \%$ \\
\hline Early ( $\geq$ 2-minutes) & 135 & $39.02 \%$ \\
\hline
\end{tabular}


Table 3

Association Between Immediate Administration of Epinephrine (within 1-minute) versus Early Administration of Epinephrine ( $\geq 2$-minutes) With Variable and Outcomes (Mean age, CPR duration, Endotracheal intubation during CPR, ROSC (>20 minutes but less than 24-hours), Survival to Admission (ROSC > 24-hours))

\begin{tabular}{|lllll|}
\hline Variable/Outcome & $\begin{array}{l}\text { Immediate (within 1- } \\
\text { minute) }\end{array}$ & $\begin{array}{l}\text { Early ( } \geq 2- \\
\text { minutes) }\end{array}$ & $\begin{array}{l}\text { P- } \\
\text { value }\end{array}$ & $\begin{array}{l}\text { Odds ratio } \\
\text { [95\% Cl] }\end{array}$ \\
\hline Mean age (in years) & 62.75 & 58.74 & 1.96 & - \\
\hline CPR Duration (in minutes) & $19: 06$ & $19: 04$ & 1.96 & - \\
\hline $\begin{array}{l}\text { Endotracheal intubation during } \\
\text { CPR }\end{array}$ & $89(63.57 \%)$ & $84(65.12 \%)$ & 0.06 & - \\
\hline $\begin{array}{l}\text { ROSC (>20-minutes but less } \\
\text { than 24-hours) }\end{array}$ & $77(36.67 \%)$ & $32(19.87 \%)$ & $<$ & $2.36[1.46-$ \\
$\begin{array}{l}\text { Survival to Admission }(>24- \\
\text { hours) }\end{array}$ & $40(14.44 \%)$ & $9(6.92 \%)$ & 0.02 & $2.26[1.06-$ \\
\hline
\end{tabular}

\section{Discussion}

Our findings reveal that immediate administration of intravenous Epinephrine is associated with increased sustained return of spontaneous circulation (ROSC) (20-minutes - 24-hours) and survival to admission. Little controversy exists over the ability of Epinephrine to increase ROSC rates. In aggregate, there is strong agreement across large number of clinical studies, that Epinephrine use improves the chances of ROSC but does not benefit survival. $[18,19,20]$ Notably, some studies suggest that Epinephrine might actually worsen neurologic outcome with increasing cumulative dose of Epinephrine. [21] Early Epinephrine administration is practically achievable in-hospital as opposed to out-of-hospital settings. Our study reveals that majority of patients received epinephrine within 1-minute of recognition (60.98\%). A study by Hansen at al. [22] conducted a secondary analysis on 26,755 patients in the out-ofhospital setting. A 10-minute cutoff for time to emergency medical services (EMS) arrival to Epinephrine administration was used. The majority received Epinephrine > 10 minutes from EMS arrival (54.2\%). The highest survival to discharge was noted when Epinephrine was given before 4 minutes, which occurred in $7 \%$ of patients. Moreover, each additional minute of time to from EMS arrival to Epinephrine was associated with $4 \%$ decrease in odds of survival to hospital dishcarge (odds ratio [OR], $0.96 ; 95 \%$ confidence interval [Cl], 0.95-0.98). However, there are profound differences between patients with in and out of hospital, in terms of patients' characteristics, underlying etiology, treatment and timing of treatment, and outcomes.

Donnino et al. [12] conducted a post hoc analysis of prospectively collected data in a large multicenter registry of in-hospital cardiac arrests (Get With The Guidelines-Resuscitation). They included 25,095 patients from 570 hospitals with asystole (55\%) or pulseless electrical activity (45\%). The median time to Epinephrine administration was three (interquartile range 2-4). Survival to 24-hours occurred in 6,280 $(27 \%)$ patients, whereas only $2,603(10 \%)$ survived to dishcarge. A stepwise decrease in survival to 
discharge with additional minute of first administration of Epinephrine: 929 (12\%) survived when epinephrine was given in the first minute, $392(12 \%)$ in the second minute, $305(11 \%)$ in the third minute, $208(9 \%)$ in the fourth minute, $335(10 \%)$ in the fifth minute, $124(10 \%)$ in the sixth minute, and $310(7 \%)$ in the seventh minute or later $(P<0.001)$. Results of our study reveal slightly higher rates of asystole (62.14\%) but less pulseless electrical activity (37.86\%). Survival to 24 -hours in our study was $30.35 \%$ of patients, which is close to results observed by Donnino et al. In our study the mean time to first Epinephrine administration was 2.86 \pm 5.06 minutes and most patients received Epinephrine within 1minute $(60.27 \%)$ of recognition of cardiopulmonary arrest. We used a cutoff of 1 -minute for first Epinephrine administration. This was used due to the time-sensitive interventions required during the lowflow state, in order to maintain coronary and cerebral perfusion without interruption. Results of this study show that immediate Epinephrine administration is associated with higher rates of ROSC (20-minutes 24-hours) (odds ratio [OR], 2.36; 95\% confidence interval [CI], 1.46-3.81) and survival to admission (odds ratio [OR], 2.26; 95\% confidence interval [Cl], 1.06-4.83) when compared with early Epinephrine. Additionally, Figs. 1 and 2 demonstrate a sharp decrease in ROSC from $36.67 \%$ when Epinephrine was administered between $0-1$ minutes to $21.21 \%$ when Epinephrine was administered between 2-3 minutes and in survival to admission from $14.44 \%$ in the $0-1$ minute group to $6.92 \%$ in the $\geq 2$ minute group. Therefore, the number of patients who need to be treated with epinephrine to achieve one patient with ROSC (20 min-24 hrs) is 6 and to achieve one patient with ROSC (>24-hours) is 13.

While the American Heart Association (AHA) recommend immediate and uninterrupted chest compressions to maintain coronary and cerebral perfusion, no strong recommendations exist in terms of timing of first Epinephrine administration nor do they recommend a maximum dose. In fact, the latest AHA guidelines recommend that Epinephrine be administered as early as feasibly possible and thereafter every 3-5 minute intervals. The physiologic rationale for early Epinephrine administration is strong. The combination of immediate high-quality chest compression and immediate Epinephrine administration could potentially result in better outcomes. Although, the results of our study encourage immediate Epinephrine administration, they question the benefit of Epinephrine after a certain amount of time.

\subsection{Limitations}

This was a retrospective analysis and therefore are only able to comment on association rather than causation. Data represents experiences from three different sites and therefore, may cause variations in results. Moreover, we did not attempt to adjust for other confounding factors such as; age, underlying diagnosis, initial rhythm, cumulative epinephrine dose, CPR metrics, time and location of arrest, etc. This may preclude the generalizability of our results.

\section{Conclusion}

Immediate epinephrine administration is associated with better rates of ROSC for up to 24-hours and beyond 24-hours for in-hospital cardiopulmonary arrests with non-shockable rhythms. This is achievable in the in-hospital setting. Therefore, we encourage initiating immediate CPR in conjunction with 
immediate epinephrine administration. Larger studies are required to find the true benefit of immediate epinephrine administration.

\section{Declarations}

\section{Ethics approval and consent to participate}

This study was approved by the Ministry of Health in Jeddah (registration no. H-02-J-002, approval no. A00440). This study was performed in accordance with the Declaration of Helsinki and the Saudi Arabian Ministry of Health research ethics committee guidelines and regulations. Waiver of informed consent was granted given the retrospective nature of the study.

\section{Consent for publication}

Not applicable

\section{Competing interests}

The authors declare that they have no competing interests

\section{Availability of data and material}

Data are available upon reasonable request from the corresponding author

\section{Funding}

This study received no funding

\section{Authors contributions}

Abdullah Bakhsh conceived, designed the study and drafted the manuscript. Maha Safhi, Ashwaq Alghamdi and Amjad Alharazi collected data and perform statistical analysis. Bedoor Alshabibi, Rajwa Alobaidi, and Maryam Alnashri analyzed the results. All authors reviewed, revised and approved the final version.

\section{Acknowledgments}

The authors would like to thank the Ministry of Health in Jeddah, Saudi Arabia for their cooperation in providing access to data.

\section{References}

1. Gough, C.J.R., Nolan, J.P. The role of adrenaline in cardiopulmonary resuscitation. Crit Care 22, 139 (2018). 
2. Overgaard CB, Dzavík V. Inotropes and vasopressors: review of physiology and clinical use in cardiovascular disease. Circulation. 2008 Sep 2;118(10):1047-56.

3. Coons JC, Seidl E. Cardiovascular pharmacotherapy update for the intensive care unit. Crit Care Nurs Q. 2007 Jan-Mar;30(1):44-57.

4. Nakahara S, Tomio J, Takahashi H, Ichikawa M, Nishida M, Morimura N, et al. Evaluation of prehospital administration of adrenaline (epinephrine) by emergency medical services for patients with out of hospital cardiac arrest in Japan: controlled propensity matched retrospective cohort study. BMJ. 2013 Dec 10;347:f6829.

5. Goto Y, Maeda T, Goto Y. Effects of prehospital epinephrine during out-of-hospital cardiac arrest with initial non-shockable rhythm: an observational cohort study. Crit Care. 2013 Sep 3;17(5):R188.

6. Dumas F, Bougouin W, Geri G, Lamhaut L, Bougle A, Daviaud F, et al. Is epinephrine during cardiac arrest associated with worse outcomes in resuscitated patients? J Am Coll Cardiol. 2014 Dec 9;64(22):2360-7.

7. Fries $\mathrm{M}$, Weil MH, Chang YT, Castillo $\mathrm{C}$, Tang W. Microcirculation during cardiac arrest and resuscitation. Crit Care Med. 2006 Dec;34(12 Suppl):S454-7.

8. Halvorsen P, Sharma HS, Basu S, Wiklund L. Neural injury after use of vasopressin and adrenaline during porcine cardiopulmonary resuscitation. Ups J Med Sci. 2015 Mar;120(1):11-9.

9. Panchal AR, Bartos JA, Cabañas JG, Donnino MW, Drennan IR, Hirsch KG, et al; on behalf of the Adult Basic and Advanced Life Support Writing Group. Part 3: adult basic and advanced life support: 2020 American Heart Association Guidelines for Cardiopulmonary Resuscitation and Emergency Cardiovascular Care. Circulation. 2020;142(suppl 2):S366-S468.

10. Sigal AP, Sandel KM, Buckler DG, Wasser T, Abella BS. Impact of adrenaline dose and timing on outof-hospital cardiac arrest survival and neurological outcomes. Resuscitation. 2019;139:182-8.

11. Nakahara S, Tomio J, Nishida M, Morimura N, Ichikawa M, Sakamoto T. Association between timing of epinephrine administration and intact neurologic survival following out-of-hospital cardiac arrest in Japan: a population-based prospective observational study.

12. Donnino MW, Salciccioli JD, Howell MD, Cocchi MN, Giberson B, Berg K, et al; American Heart Association's Get With The Guidelines-Resuscitation Investigators. Time to administration of epinephrine and outcome after in-hospital cardiac arrest with non-shockable rhythms: retrospective analysis of large in-hospital data registry. BMJ. 2014 May 20;348:g3028.

13. Andersen LW, Berg KM, Saindon BZ, Massaro JM, Raymond TT, Berg RA, et al; American Heart Association Get With the Guidelines-Resuscitation Investigators. Time to Epinephrine and Survival After Pediatric In-Hospital Cardiac Arrest. JAMA. 2015 Aug 25;314(8):802-10.

14. Khera R, Chan PS, Donnino M, Girotra S; American Heart Association's Get With The GuidelinesResuscitation Investigators. Hospital Variation in Time to Epinephrine for Nonshockable In-Hospital Cardiac Arrest. Circulation. 2016 Dec 20;134(25):2105-2114.

15. Bircher NG, Chan PS, Xu Y; American Heart Association's Get With The Guidelines-Resuscitation Investigators. Delays in Cardiopulmonary Resuscitation, Defibrillation, and Epinephrine 
Administration All Decrease Survival in In-hospital Cardiac Arrest. Anesthesiology. 2019 Mar;130(3):414-422.

16. Perkins GD, Ji C, Deakin CD, Quinn T, Nolan JP, Scomparin C, et al; PARAMEDIC2 Collaborators. A Randomized Trial of Epinephrine in Out-of-Hospital Cardiac Arrest. N Engl J Med. 2018 Aug 23;379(8):711-721.

17. Jung J, Rice J, Bord S. Rethinking the role of epinephrine in cardiac arrest: the PARAMEDIC2 trial. Ann Transl Med 2018;6(Suppl 2):S129.

18. Olasveengen TM, Sunde K, Brunborg C, Thowsen J, Steen PA, Wik L: Intravenous drug administration during out-of-hospital cardiac arrest: A randomized trial. JAMA 2009; 302:2222-9

19. Jacobs IG, Finn JC, Jelinek GA, Oxer HF, Thompson PL: Effect of adrenaline on survival in out-ofhospital cardiac arrest: A randomised double-blind placebo-controlled trial. Resuscitation 2011; 82:1138-43

20. Stiell IG, Wells GA, Field B, Spaite DW, Nesbitt LP, De Maio VJ, et al; Ontario Prehospital Advanced Life Support Study Group: Advanced cardiac life support in out-of-hospital cardiac arrest. N Engl J Med 2004; 351:647-56

21. Arrich J, Sterz F, Herkner H, Testori C, Behringer W: Total epinephrine dose during asystole and pulseless electrical activity cardiac arrests is associated with unfavourable functional outcome and increased in-hospital mortality. Resuscitation 2012; 83:333-7

22. Hansen M, Schmicker RH, Newgard C, Grunau B, Scheuermeyer F, Cheskes S, et al. Time to Epinephrine Administration and Survival From Nonshockable Out-of-Hospital Cardiac Arrest Among Children and Adults. Circulation 2018; 138(19):2032-2040

\section{Figures}




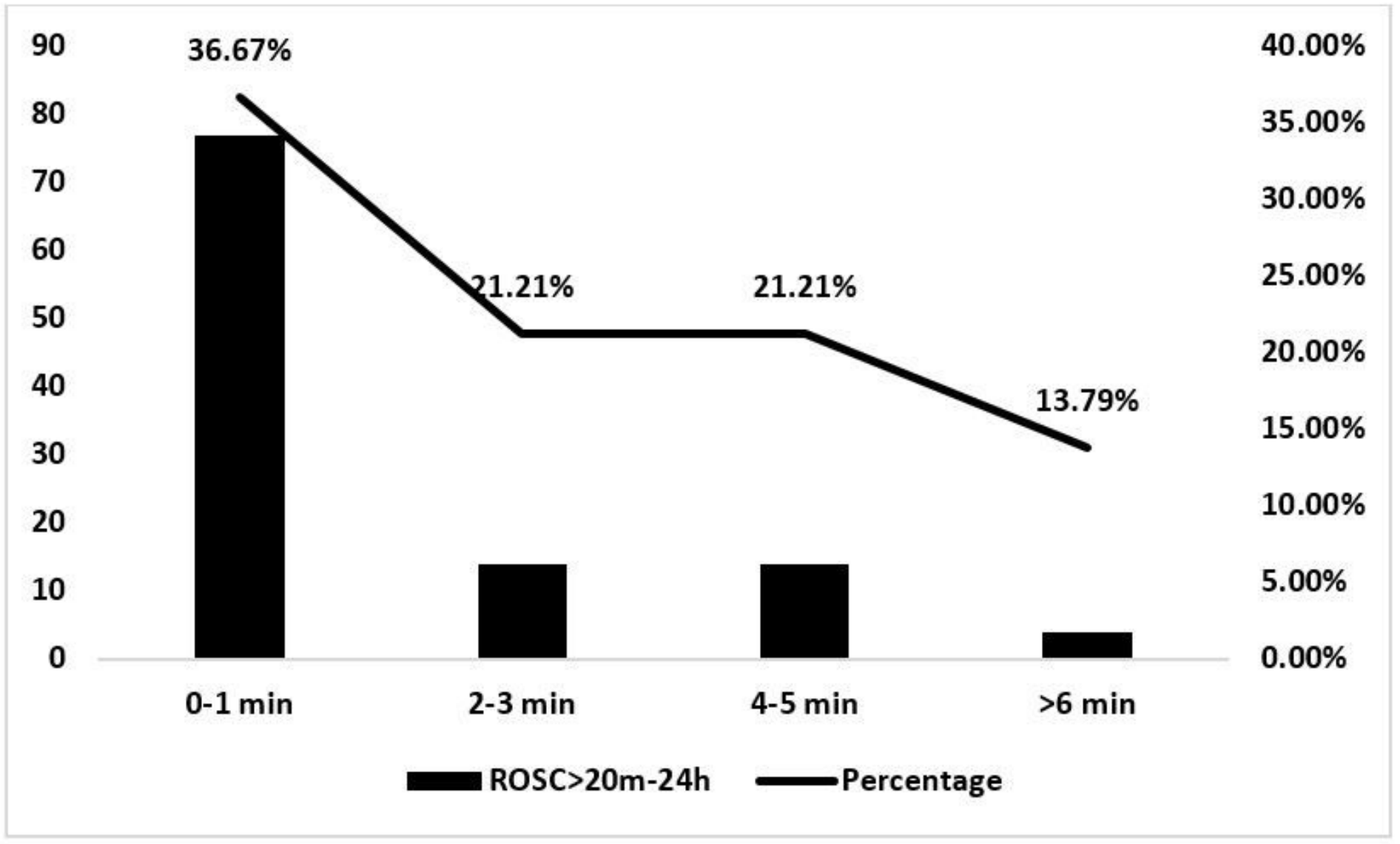

Figure 1

Association Between Timing of First Dose of Epinephrine With Sustained Return of Spontaneous Circulation ( $\geq 20$-minutes but $<24$-hours) 


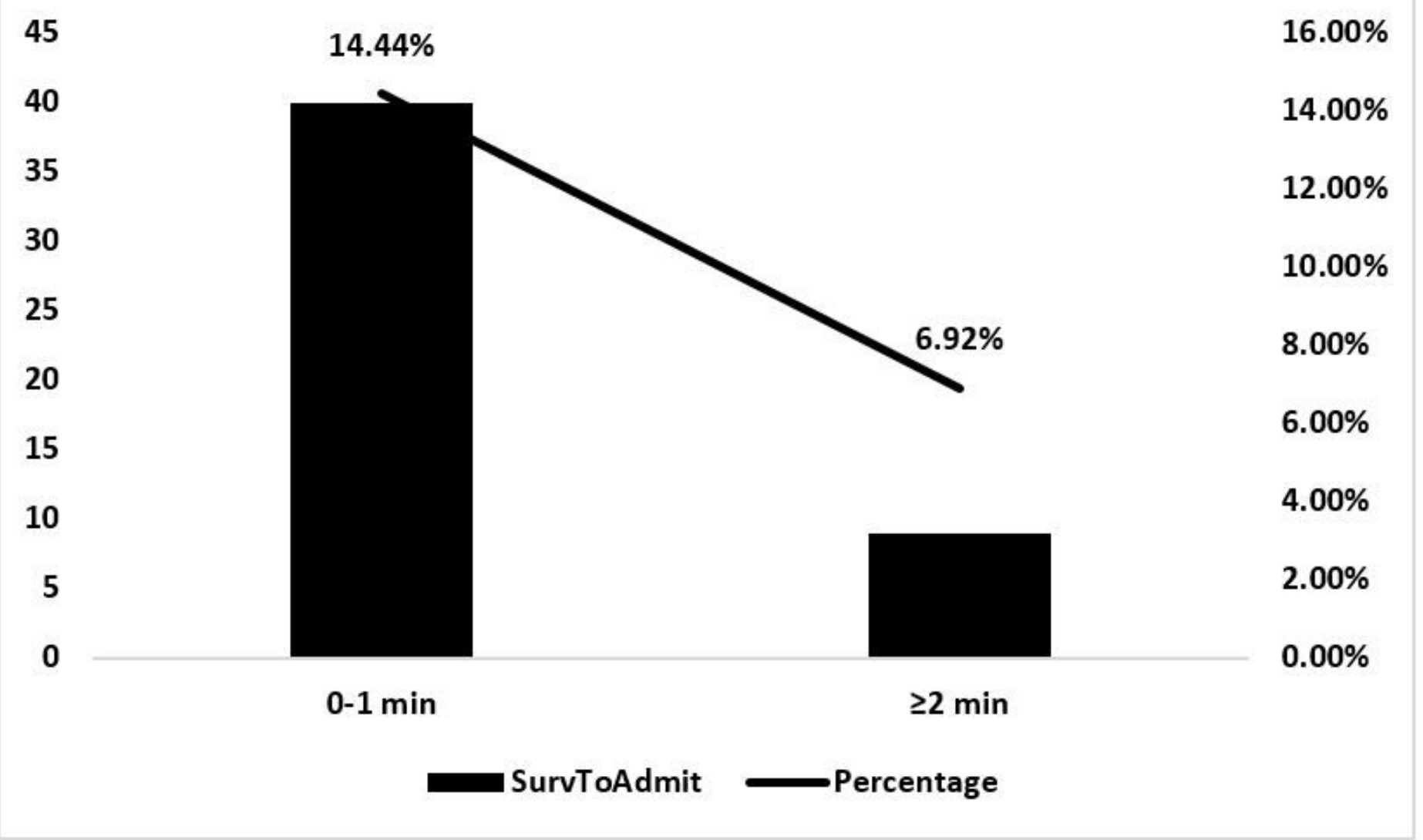

Figure 2

Association Between Timing of First Dose of Epinephrine With Survival to Admission 